merely recount them without making a single remark. The instruments introduced were the following :-

1. An improved aneurism needle, manufactured by Messrs. Hutchinson, of Sheffield.

2. A new Italian toumiquet, the object of which is to compress only the main trunk of the blood-vessels of the extremity without encircling the entire limb.

3. An improved patent truss, by the inventor, $\mathrm{Mr}$. Alfred Greene.

The following morbid specimens were exhibited :-

1. The upper portion of the femur, the neck of which had been fractured five weeks previously, and in which there was no attempt at reunion, by $\mathrm{Mr}$. Overend.

2. The heart of a child, in which the posterior inferior surface of the right ventricle was perforated-the pericardium was also perforated, and at the time of the post-mortem examination was filled with blood, as was also the right pleura; a needle, about an inch in length, and dark-coloured, was lying on the diaphragm, and had doubtless caused the perforations-by $\mathrm{Mr}$. Overend.

3. Portion of the bone of the leg of a horse which had died of traumatic tetanus, by Mr. H. Jackson.

4. Necrosis and exostosis of the maxillary bone of a horse, resulting from a violent blow, by $\mathrm{Mr}$. $\mathrm{H}$. Taylor, V.S.

5. Lungs of a horse thickly studded with tubercles, by Mr. H. Taylor, V.S.

6. Fracture of several of the dorsal vertebræ, with considerable displacement, the consequence of a fall, by $\mathrm{Mr}$. Thomas.

7. Bony tumor found in the pons Varolii of a female who died of puerperal convulsions, by Mr. Thomas.

8. Imperfectly closed foramen ovale, by Dr. Favell.

9. Scirrhous omentum, by Dr. Favell.

19. Perforation of the stomach, by Dr. Favell.

11. Medullary sarcoma of the uterus, by Dr. Favell.

12. Granular degeneration of the kidney, by Dr. Favell.

13. Tubercular liver, by Dr. Favell.

14. Extensive hypertrophy of the heart, with adhesion of the pericardium, and perforation of one of the semilunar valves of the aorta, by Dr. Favell.

15. The heart of a child, aged eight, in which there was only one ventricle; the foramen ovale was perfectly unclosed, and the septum of the heart was perforated by a large opening, which allowed the blood to pass from the left ventricle into the commencement of the pulmonary artery, by Dr. Favell.

16. Portion of the intestine of a child, the serous coat of which was thickly studded with tubercles, by Dr. Favell.

I must further observe that, during the session, $\mathrm{Mr}$. H. Jackson placed on the table some drawings of morbid parts under his care at the infirmary, and also several rare and interesting medical works.

It is almost superfluous for me, in conclusion, to remark that the communications, the discussions, and the number and variety of the exhibitions which have been introduced during the session, have greatly exceeded the expectation of those who were the founders of the society. We may look back upon the past with perfect satisfaction, and it certainly is not the least pleasing part of our retrospect to be able to assert that, during the entire session, not one unkind word, not a single harsh phrase, not a single sentiment has been uttered which could possibly occasion pain or uneasiness to the most delicate and sensitive mind. I will only add the expression of my fervent hope, that, when we meet again, it may be in the same kindliness of spirit, with similar animating zeal, and with the same single aim, not to minister to our individual selfishness, but to contribute our share towards the scientific advancement of our common profession.

\section{CASE OF FRACTURE}

OF THE

\section{FIFTH AND SIXTH CERVICAL VERTEBRA}

\section{By Alfred Baker, Esq}

[Read before the Pathological Society of Birmingham.]

William Bache, a robust, florid, muscular, and well developed carpenter, a single man, was brought to the hospital on the 23rd of April, 1842, at half past eleven, a.m., in consequence of his having fallen from the kitchen into the cellar of a house where he was at work. He states that he fell head foremost, and was found lying with his head downwards. The height was about seven feet, and the accident occurred a quarter of an hour ago.

Present State.-He complains of severe pain in the back of the neck, which is much aggravated by the motions of the head, and by raising him into the semierect posture. The neck is swollen, especially on either side of the spinal column, and a crepitation, like that from a synovial fluid is distinguishable here on flexing the head. Another crepitus, more like that of bone, is perceived just over the fifth and sixth cervical spines, whilst performing the same movements; but I could not positively grasp and localise any movable portion. He has headache; his pupils are of moderate size and activity; he has a total abolition of motion and sensitive power in the lower extremities and abdomen, and these parts may be pinched without his being cognisant of the act. Upon ticklin the soles, one or two of the toes were slightly flexed, but the spasm ceased immediately; his penis is in a state of rigid priapism, but is quite devoid of sensation; the upper extremities are also paralysed, but the sensitive fibres of the nerves have sustained more injury than the motor, since he can raise the limbs, whilst he complains of want of feeling and numbness in them, and does not suffer from pinching; he has also shortness of breath, but his voice is sonorous; has dull aching pain in both eyebrows and in the forehead; pain in neck, and upper part of shoulders, increased by moving his body. The priapism lasted about three hours, since which time it has been less marked, the organ being semi-erect and tumid; the scrotum is injected. The bowels have been moved twice by an enema, but he has voided no urine, and the hypogastrium is swollen and tense; the conjunctivæ are injected; the face is deeply-coloured; lips dry ; he is thirsty ; has a whitish and moist tongue, which he protrudes in the median line; his eyesight and hearing are acute. Nearly three pints of urine were drawn off by the catheter.

24. Had very little sleep, and lies upon his right side, in which posture he is more easy than when 
supine, when he feels pain in the neck; his face is ruddy, and does not express pain; his respiration is not so laboured; the abdominal muscles are raised me and depressed whilst breathing, but this arises probably from the mere depression and elevation of the diaphragm in these acts; his voice is still loud; pulse soft, distinct, and 66 per minute; skin warm and moist; the lower extremities and trunk are still quite paralysed, but he retains sufficient sensation to distinguish between warmth and cold, being cognisant of the warmth of some water used to wash his feet this morning, at the same time he is insensible to pinching; the penis remains swollen and semi-erect; he has more sensation in the upper extremities; is aware when either thumb is pinched, but if the fingers be grasped, says he feels something in the middle of his arm; his urine is still retained and has been drawn off by a catheter; his bowels also have been slightly moved without volition or cognisance.

20. Sleeps but little, and seems to be gradually sinking; his intellectual faculties remain unimpaired; pulse 80 , soft and feeble; skin warm; breathing more difficult, irregular, and hurried; his voice is to day less sonorous; takes very little food; the paralysed parts seem to be in the same condition.

27 . Is evidently sinking; his face is tumid and purple; pupils small and active; senses perfect and intellect clear ; voice weaker; respiration laborious, irregular, and attended with loud, coarse tracheal, and bronchial rattles; fulse 76 ; palsy increased; has passed a stool unconsciously with a little urine having an ammoniacal odour.

28. Passed a restless night, dyspnœa being severe; he rallicd for an hour this morning, but soon became worse, and expired at ten, a.m., having continued intelligent to the last.

Sectio Cadaveris, Forty-eight Hours after Death.

Head.-Meninges healthy; no unusual injection of the vessels of the pia mater; brain itself of good consistence, though somewhat congested; the ventricles were all normal; they contained each one ounce of bloody serum, the results probably of transudation after death ; the posterior lobes of the brain were more congested than the anterior; the velum interpositum and choroid plexuses were somewhat turgid, but the sinuses were not engorged; the skull itself had sustained no appreciable injury.

Spine.-The vertebra, when the subject was laid on the belly, and the dorsal muscles detached, were found to be on a natural and equal plane, and there was no divergence from the median line; the fifth and sixth cervical vertebra, when grasped, were found fractured on both sides of the arch, near the point where the lamina unite with the oblique processes, but the fragments were not completely detached, the periosteum not being turn quite through, so that no displacement occurred. 'The body of the fifth, when the chord was removed, was seen to be broken through in the centre, but here also the clcavage was incompletc, and the ligaments, not being lacerated, no displaccment had resulted; a quantity of dark coloured fluid blood surrounded the investments of the spinal chord in the lumbar and cervical regions, and the membranes were stained in the other parts, as though the blood had gravitated from above; the membranes had suffered no laceration, but at athe point opposite the injured vertebrx the chord seemed bulging, and enlarged more than is usual, and the cellular membrane next to the sheath was infiltrated with blood. These membranes exhibited no traces of inflammatory action; the pia mater was somewhat congested below the seat of injury; when the membranes were divided, and the spinal medulla cut open, it was seen to have a normal appearance, both above and below the expanded portion, its colour being natural and its consistence firm; that part, however; corresponding to the fifth and sixth cervical vertebre was soft, creamy, almost diffluent, and had a purplish red colour, of different depths in different portions; at first sight, the whole of this had a confused look, as though the whole substance of the medulla had been disintegrated by sanguineous effusion, and was mixed and blended with it, but upon careful examination, a few fibres of the chord on its external and posterior surface might be traced onwards, as though maintaining continuity between the upper and lower segments, but even here, small maculæ of red colour might be discovered, interrupting and breaking the white colour; at one or two points, clots of blood distinctly separable from the medullary matter were visible; the whole morbid space was tolerably defined in its boundaries; a small coagulum was also found exteriorly within the arachnoid cavity, em. bracing the roots of one of the left dorsal (first) nerves immediately below the seat of injury; the medulla oblongata and nerves arising from it presented no abnormal appearances.

\section{ENCYSTED URINARY CALCULUS IN A CHILD.} TO THE EDITORS OF THE PROVINCIAL MEDICAL
JOURNAL.

Gentlemen, - If you think the following case worthy of being recorded in your useful periodical, it will much oblige

\author{
Yours, faithfully, \\ JAMES THEODORICK VALE, M.R.C.S.L.
}

Birkenhead, Cheshire, June 16, 1812.

B., a healthy-looking girl, about three years and a half old, has suffered nearly twelve months with a frequent disposition to urinate, accompanied with pain in voiding it, which was increased when the bladder was empty; bowels often in a disturbed state; prolapsus ani generally occurs when the bowels are acted on; she never takes any exercise, or plays with her companions; the fingers of one hand are constantly applied to the genital organs, the seat of her sufferings being at the end of the urethra; there is seldom any alleviation from pain, excepting when nursed on her mother's knee, or rocking herself in a chair, in which she sits the greater part of the day; there is sometimes incontinence of urine, which exhales a strong, offensive, alkaline odour, destroying the linen and bed-clothes; no blood passes from the viscus, but the urine is occasionally turbid. On sounding the bladder, a stone was instantly struck, emitting a peculiar dull sound.

May 5 . In the presence of a few medical friends, a sinall dilator oris was insinuated into the meatus 